\title{
INFLUÊNCIA DO TAMANHO DE SEMENTES NA GERMINAÇÃO E VIGOR INICIAL DA SOJA (Glycine max)
}

\author{
Luciana de Oliveira Derre ${ }^{1}$, Jean Arciso Daltoé ${ }^{1}$, Vania Sarubo ${ }^{1}$, Fabiana Lima Abrantes ${ }^{2}$ \\ ${ }^{1}$ Faculdade Centro Mato-Grossense - FACEM, curso de Agronomia, Sorriso, MT. ${ }^{2}$ Universidade do Oeste Paulista - \\ UNOESTE, Presidente Prudente, SP. E-mail: luciana derre@outlook.com
}

\begin{abstract}
RESUMO
A cultura da soja tem grande importância para a agricultura, e para um bom estabelecimento inicial da lavoura, as sementes estão entre os insumos mais importantes. O objetivo deste trabalho foi avaliar a germinação e vigor inicial de sementes de soja de diferentes tamanhos. $O$ trabalho foi realizado no Laboratório de Análises de Sementes da Faculdade Centro MatoGrossense (FACEM), onde foram analisadas sementes da variedade M8372IPRO em diferentes diâmetros. Utilizou-se o delineamento inteiramente casualizado, sendo 4 tratamentos: 3 tamanhos de peneiras $(5,5 ; 6,0 ; 6,5)$ e Amostra de Campo (sem classificação de peneiras), com cinco repetições. Avaliou-se a germinação, teste de tetrazólio, IVG, comprimento de plântulas e de raiz. Sementes maiores apresentaram maior qualidade fisiológica e maiores taxas de germinação. O tamanho das sementes não interferiu significativamente no comprimento de plântulas e de raízes. Concluímos que diâmetros maiores de sementes promovem maior vigor e maiores taxas de germinação, mas o desenvolvimento da raiz e parte aérea não foi influenciado.
\end{abstract}

Palavras chave: semente, qualidade fisiológica, tetrazólio, classificação por peneiras, desenvolvimento,

\section{INFLUENCE OF SEED SIZE IN THE GERMINATION AND INITIAL VALIDITY OF SOYBEANS (Glycine max)}

\section{ABSTRACT}

Soybean cultivation is of great importance for agriculture, and for a good initial establishment of the crop, seeds are among the most important inputs. The objective of this work was to evaluate the initial germination and vigor of soybean seeds of different sizes. The work was carried out in the Laboratory of Seed Analysis of the Faculdade Centro Mato-Grossense (FACEM), where seeds of the variety M8372IPRO were analyzed in different diameters. A completely randomized design was used, with 4 treatments: 3 sieve sizes $(5.5,6.0,6.5)$ and Field Sample (without sieve classification), with five replications. Germination, tetrazolium test, IVG, seedling and root length were evaluated. Larger seeds showed higher physiological quality and higher germination rates. Seed size did not significantly affect seedling and root length. We concluded that larger seed diameters promote greater vigor and higher germination rates, but the development of root and shoot was not influenced

Key words: seed, physiological quality, tetrazolium, classification by sieves, development

\section{INTRODUÇÃO}

Dentre as espécies produtoras de grãos, cultivadas no Brasil, a soja (Glycine max) é considerada uma das culturas de maior potencial econômico para a comercialização interna e externa. É também uma das mais importantes oleaginosas do mundo. Nos últimos anos, principalmente com a abertura de novas áreas sob vegetação de cerrado, o Brasil passou a ser um 
importante produtor de soja (WAYNER ÁVILA et. Al. 2008). A cultura tem grande importância no contexto agrícola de produção brasileira e mundial, representando aproximadamente $48,63 \%$ da produção nacional de grãos (CONAB, 2016). Essa produção expressiva está atrelada ao manejo adequado da cultura e ao uso cada vez maior de sementes de alta qualidade (SOUZA 2007).

A semente é considerada o mais importante insumo agrícola, pois, conduz ao campo as características genéticas determinantes do desempenho da cultivar e ao mesmo tempo é responsável por contribuir decisivamente para o sucesso do estabelecimento do estande desejado, fornecendo a base para produção rentável (MARCOS FILHO, JULIO; 2005).

A importância do tamanho das sementes tem sido relatada em uma série de artigos, como. Lima, 1996; Beckert et al., 2000 Pelo fato de constituir um dos fatores que podem influenciar a germinação e o vigor das plântulas. As sementes de maior tamanho, que apresentam maior densidade, durante seu desenvolvimento foram as mais nutridas. Este fato torna-se mais patente nas plantas cujas sementes não são formadas todas ao mesmo tempo, de sorte que as últimas a se desenvolverem são, normalmente, menores, ou de menor densidade. Por este motivo, as maiores e as de maior densidade são as que possuem, normalmente, embriões bem formados e com maiores quantidades de reservas sendo, potencialmente, as mais vigorosas e com maior capacidade de sobrevivência. Assim, é razoável que uma cultura apresente, em condições de campo, populações de plantas diferentes em favor das sementes maiores. (CARVALHO e NAKAGAWA, 2000). No entanto, persistem as evidências de que sementes menores podem trazer economia no momento da semeadura (ANDRADE et al., 1997).

O presente trabalho teve por objetivo verificar a influência do tamanho das sementes na germinação e na viabilidade inicial de sementes de soja, classificadas por tamanhos, em relação das que não tem classificação.

\section{METODOLOGIA}

O presente trabalho foi realizado no Laboratório de Análise de Sementes da Faculdade Centro Mato-Grossense - FACEM, na cidade de Sorriso Mato Grosso.

Foram utilizadas sementes das cultivares de soja M8372IPRO da safra 2015/2016, adquirida da empresa J\&H Sementes, classificada nas peneiras de crivos circulares, de diâmetros $P$ $5,5 \mathrm{~mm}, P$ 6,0mm e P 6,5 mm além da A.C (amostra de campo) sem classificação de tamanho, as quais constituíram os tratamentos. Foram avaliadas características como, germinação, comprimento de plântulas, comprimento de raiz, teste de viabilidade, índice de velocidade de germinação e emergência.

Realizado em rolo de papel germitest, após sete dias foi realizada a contagem de plântulas normais, anormais e sementes que não germinaram segundo critérios de avaliação estabelecidos nas Regras para Análise de Sementes, sendo os resultados expressos em porcentagem. Para isso foi preparado o substrato, utilizando três folhas de papel para germinação com duas folhas na base e uma para cobrir as sementes, as quais foram enroladas no sentido da esquerda para a direita formando rolinhos, que foram colocados em sacos plásticos devidamente identificados e levados ao germinador, com temperatura constante de $25^{\circ} \mathrm{C}$ e fotoperíodo de 12 (doze) horas. A quantidade de água utilizada para umedecer as folhas foi de 2,5 vezes a massa do papel seco. As leituras foram realizadas aos 05 (cinco) e 08 (oito) dias após a semeadura (BRASIL, 2009).

A avaliação do desenvolvimento de plântulas e raiz foi realizada de acordo com Nakagawa (1999), utilizando quatro repetições de 10 (dez) sementes, que foram distribuídas com o hilo voltado para a parte inferior do papel, sobre uma linha traçada no terço superior no sentido longitudinal, por 5 dias, seguindo-se os procedimentos descritos para o teste de germinação. As plântulas normais obtidas foram avaliadas por comprimento do hipocótilo e da raiz primária. 
O índice de velocidade de germinação (IVG) foi determinado a partir do número de plântulas normais $(G)$ contadas nas leituras de germinação, dividido pelo número de dias da semeadura até a contagem (N), utilizando a fórmula IVG=G/N, segundo Nakagawa (1999).

Para determinar a viabilidade das sementes, o teste de tetrazólio foi realizado com 200 (duzentas) sementes para cada tratamento, que foram pré-condicionadas em papel toalha umedecidas com quantidade de água equivalente a 2,5 vezes a massa do papel seco, durante 16 horas, à temperatura média de 25으.

Após este período, as sementes foram colocadas em um becker, imersas em uma solução de concentração de $0,075 \%$ de 2,3,5-trifenil-cloreto-de-tetrazólio (TCT) e, em seguida, mantidas no escuro, em temperatura entre $35^{\circ}$ a $40^{\circ} \mathrm{C}$, por três horas, para o desenvolvimento da coloração. Após esse período as sementes foram lavadas em água corrente e avaliadas conforme metodologia descrita por França Neto et al. (1998).

Quando a semente de soja é imersa na solução incolor de TCT, esta é difundida através dos tecidos, ocorrendo nas células vivas a reação de redução que resulta na formação de um composto vermelho, conhecido por trifenilformazan, qual não ocorre nos tecidos mortos. Essa coloração vermelha indica que há atividade metabólica nas mitocôndrias, portanto existe viabilidade celular nos tecidos.

As diversas características foram avaliadas e conduzidas com cinco repetições de cada tratamento. As médias foram comparadas pela análise de variância, através do teste de Tukey a $5 \%$ de probabilidade.

\section{RESULTADOS}

De acordo com a comparação de médias pelo teste de Tukey a nível de $5 \%$ de probabilidade, para o tetrazólio, em relação aos diferentes tratamentos e diferentes diâmetros da semente de soja,(Figura 1), o teste foi significativo para os tratamentos com diâmetros de 5,5; 6,0 e 6,5mm. O tratamento A.C (amostra de campo) não mostrou diferença significativa em relação aos demais tratamentos.

Pode-se observar na Figura 1, que o tratamento de peneiras 6,0 e 6,5mm demonstraram a melhor viabilidade das sementes, não apresentando muitos danos fisiológicos, o qual demostrou viabilidade de germinação de $96,4 \%$ e $95,6 \% \%$. A A.C apresentou viabilidade germinativa de $95,2 \%$. Já o tratamento com peneiras $5,5 \mathrm{~mm}$ apresentaram viabilidade germinativa de $89,6 \%$, sendo a que mais apresentou danos fisiológicos.

Figura 1 - Valores médios de viabilidade de sementes obtido através do teste de tetrazolio, referente a diferentes diâmetros de semente. Sorriso. MT. 2016.

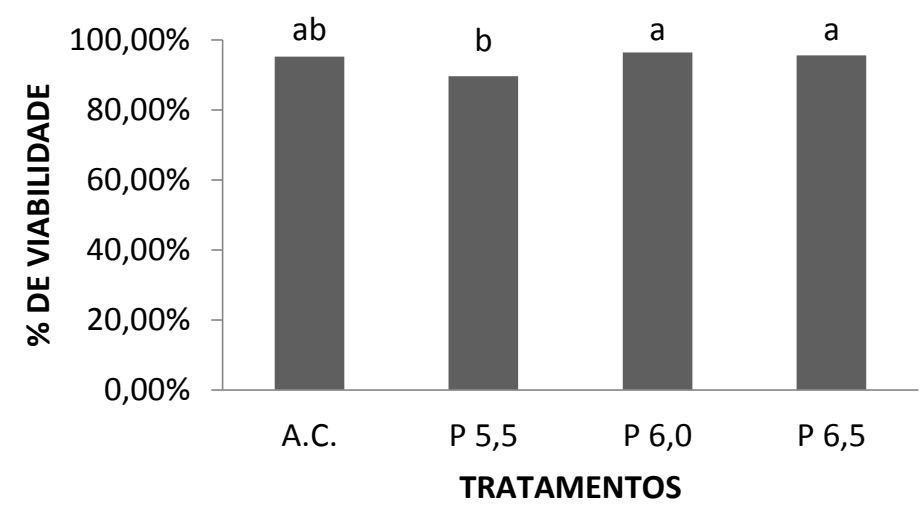

De acordo com o teste de Tukey a nível de $5 \%$ de probabilidade, para analise de germinação, em relação aos diferentes tratamentos e diferentes diâmetros da semente de 
soja,(Figura 2), o teste foi significativo para o tratamento $P$ 6,5. O tratamento A.C e $P$ 5,5 mostraram diferença significativa em relação ao tratamento $P$ 6,5. Observa-se que a germinação entre os tratamentos, sendo que o tratamento $P$ 6,5 apresentou 95,20\% de germinação, 0 tratamento $P 6,0$ apresentou germinação de $92,40 \%$, e os tratamentos P 5,5 e A.C apresentaram $88,0 \%$ e $86,8 \%$, sendo o tratamento $P$ 6,5 o melhor tratamento em germinação do que os de menores diâmetros, ou seja, conforme o diâmetro da semente era menor a germinação diminuiu graduadamente.

Figura 2 - Valores médios de germinação obtido através do teste de germinação, referente a diferentes diâmetros de semente. Sorriso. MT. 2016.

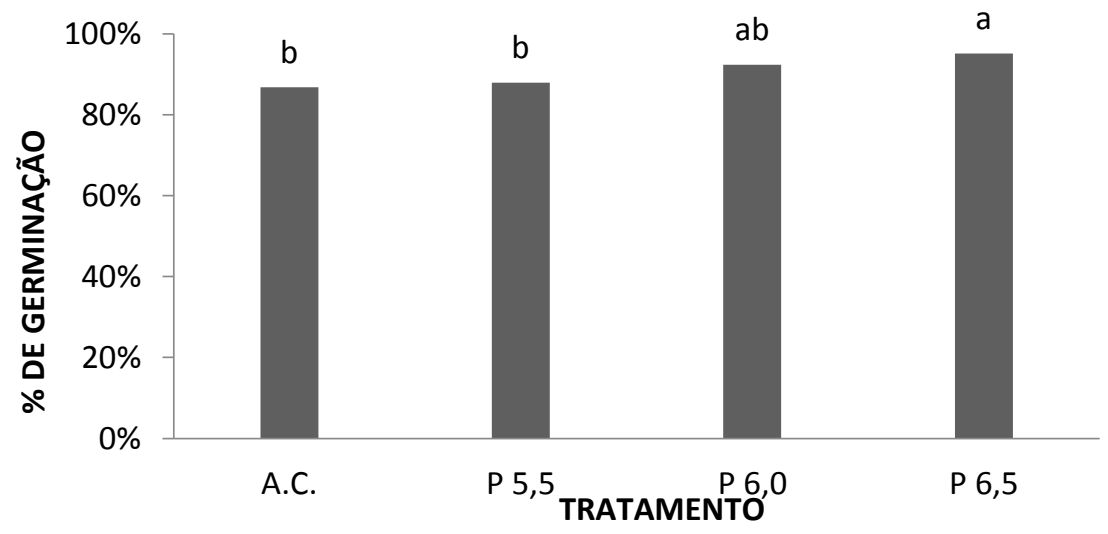

Observa-se na Figura 3, faz um comparativo entre a relação de percentual de germinação levado em consideração o resultado do teste de germinação em comparação ao potencial expresso pelo teste de tetrazólio. Observa-se que o tratamento A.C. teve uma redução de $8,4 \%$ quando comparado a germinação com o potencial germinativo expresso no teste de tretazólio, sendo o tratamento que mais apresentou perda na germinação, o tratamento $P$ 6,0 mostrou uma redução de $4 \%$ do potencial germinativo, os tratamentos $P$ 5,5 e P 6,5 apresentaram redução de $1,6 \%$ e $0,4 \%$, sendo o tratamento $P$ 6,5 o que teve melhor desempenho na germinação das sementes.

Figura 3 - Relação de germinação obtida pelo teste de germinação e a viabilidade obtido pelo teste de tetrazólio, referente a diferentes diâmetros de semente. Sorriso. MT. 2016.

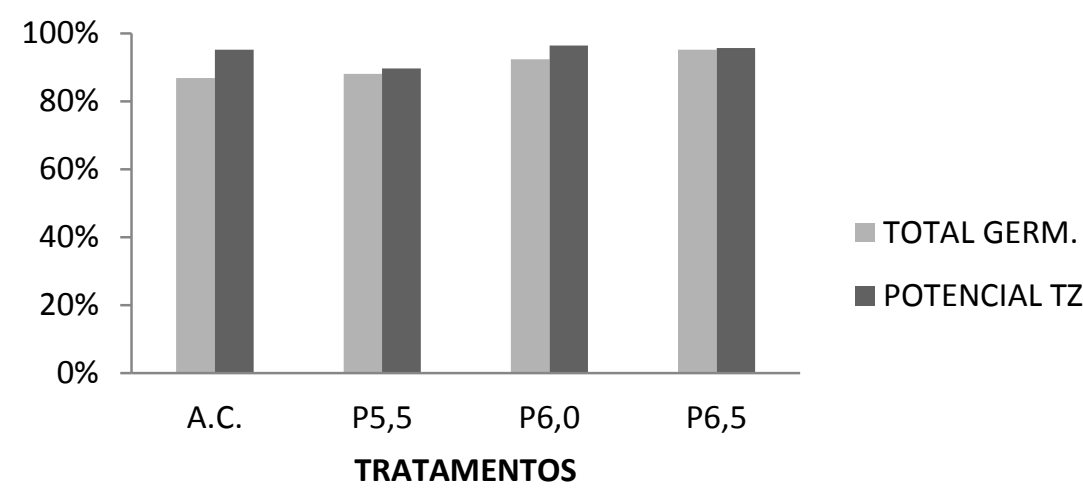

O teste de índice de velocidade de germinação pelo teste de Tukey a nível de $5 \%$ de probabilidade, mostra que os tratamentos $P$ 5,5 e P 6,0 não tiveram diferença significativa. Entre 
os tratamentos $P$ 6,5 e A.C. houve diferença, o tratamento $P$ 6,5 mostrou um acelerado índice de germinação quando comparado ao tratamento A.C, enquanto os tratamentos $P$ 5,5 e $P$ 6,0 mantiveram o mesmo índice de desenvolvimento, na figura 4 pode-se observar que a velocidade da germinação aumentou conforme o tamanho da semente. Observasse que quanto maior o diâmetro da semente a o índice de velocidade de germinação aumenta.

Figura 4 - Índice de velocidade de germinação, referente a diferentes diâmetros de semente. Sorriso. MT. 2016.

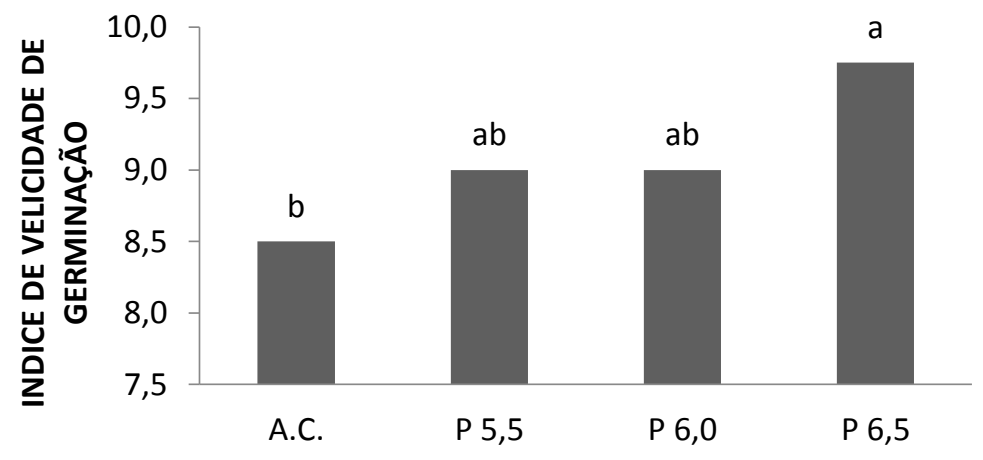

TRATAMENTOS

De acordo com a análise da variância, pelo teste de Tukey a nível de $5 \%$ de probabilidade, para avaliação de sementes mortas, (Figura 5), o teste foi significativo para os tratamento P 6,5; mostrando uma menor quantidade de sementes mortas no tratamento $P$ 6,5 mostrando $1,20 \%$, o tratamento $P$ 6,0 mostrou seu resultado não significativo pela analise de variância, com 1,6\% de sementes mortas, o tratamento $P$ 5,5 também não mostrou resultado significativo porem já teve um aumento no seu número de sementes mortas sendo de $4,40 \%$, já o tratamento A.C, mostrou diferença significativa levando o percentual de sementes mortas a 5,20\% como podemos analisar pela Figura 5.

Figura 5 - Percentagens de sementes mortas obtidas através do teste de germinação, referente a diferentes diâmetros de semente. Sorriso. MT. 2016.

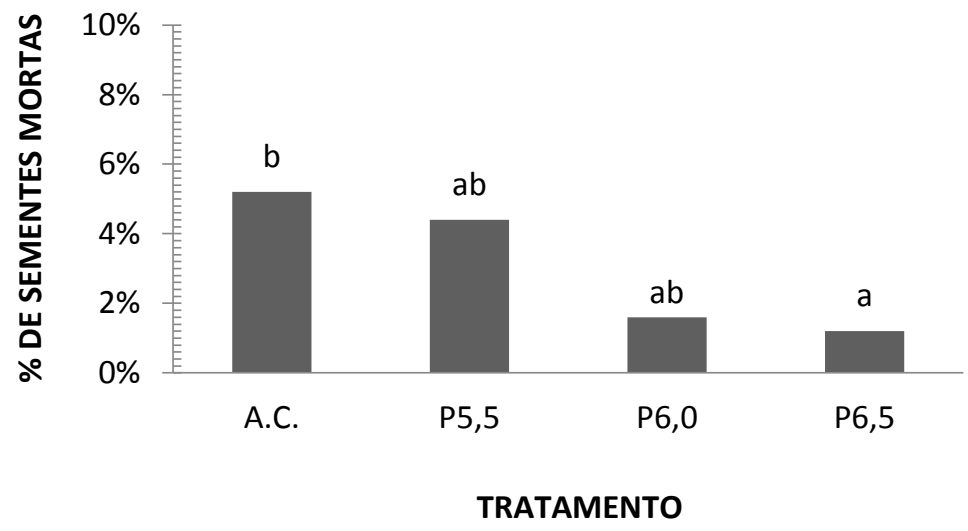

De acordo com a análise da variância, realizado pelo teste de Tukey a nível de $5 \%$ de probabilidade, os resultados obtidos na avaliação de plântulas por meio do comprimento total, dos quatro tratamentos para avaliação de sementes anormais, comprimento de plântulas e 
comprimento de raiz não mostraram diferença significativa entre os tratamentos. Como mostra a Tabela 1.

Tabela 1. Médias de comprimento de plântulas e raízes, referente aos diferentes diâmetros de sementes.

Sorriso. MT. 2016.

\begin{tabular}{ccc}
\hline & \multicolumn{2}{c}{ MEDIAS $(\mathrm{cm})$} \\
\cline { 2 - 3 } tratamentos & C. PLANTULAS & C. RAIZ \\
A.C. & $4,500000 \mathrm{a}$ & $11,250000 \mathrm{a}$ \\
P 5,5 & $4,500000 \mathrm{a}$ & $12,000000 \mathrm{a}$ \\
P 6,0 & $5,250000 \mathrm{a}$ & $12,250000 \mathrm{a}$ \\
P 6,5 & $5,250000 \mathrm{a}$ & $12,500000 \mathrm{a}$ \\
\hline
\end{tabular}

Médias seguida de letras iguais não diferem entre si, pelo teste de Tukey a $5 \%$ de probabilidade.

\section{DISCUSSÃO}

Segundo Vinhal-Freitas (2011), há um efeito significativo do tamanho de sementes em relação ao vigor, onde sementes de soja com maiores diâmetros demonstram maior capacidade fisiológica.

Segundo Franca Neto (1998), o teste de tetrazolio e forma mais rápida e segura de se avaliar viabilidade das sementes, com base na coloração dos tecidos dos grãos. A coloração indica a atividade respiratória nas mitocôndrias, permitindo identificar o tecido que respira (vivo) e o que apresenta atividade fisiológica deficiente, pois este permanece descolorido ou exibe coloração anormal (MARCOS FILHO, 2005).

Segundo o trabalho realizado por Pádua et. Al. (2010), diz que sementes de maiores diâmetros mostram que a germinação e vigor são superior em relação a sementes menores.

\section{CONCLUSÃO}

Com o presente trabalho e possível concluir através de análises estatísticas que sementes de diâmetros maiores podem ter interferência significativa em germinação, no índice de velocidade de germinação (IVG) e no vigor inicial.

\section{REFERÊNCIAS}

ANDRADE, R.V.; ANDREOLI, C.; BORBA, C.S.; AZEVEDO, J.T.; MARTINS-NETTO, D.A. \& OLIVEIRA, A.C. Efeito da forma e do tamanho da semente no desempenho no campo de dois genótipos de milho. Revista Brasileira de Sementes, Brasília, v.19, n.1, p.62-65, 1997. https://doi.org/10.17801/0101-3122/rbs.v19n1p62-65

BARZOTTO, Flávia; FACCO, Leonardo; MATTIONI, Nilson; FARIAS, G. Julia; SEGALIN, Samantha. Resposta De Cultivares De Soja À Germinação Sob Temperaturas Sub Ótimas, 2012, disponível em: www.unifra.br/eventos/sepe2012/Trabalhos/5625.pdf. Acesso: 27/10/15.

BRASIL. Regras para análise de sementes / Ministério da Agricultura, Pecuária e Abastecimento. Secretaria de Defesa Agropecuária. - Brasília : Mapa/ACS, 2009. 
CARVALHO, N.M.; NAKAGAWA, J. Sementes: ciência, tecnologia e produção. Funep: Jaboticabal, 2000. 588p.

CONAB, Companhia Nacional de Abastecimento, Acompanhamento da safra brasileira de grãos, v. 9 Safra 2015/16 - Nono levantamento, Brasília, ISSN 2318-6852 p.-174, junho 2016.

COSTA, Nilton Pereira; MARCOS FILHO, Julio; FRANÇA NETO, José de Barros; KRZYZANOWSKI, Francisco Carlos; HENNING, Ademir Assis; Teste de tetrazólio em semente de soja com precondicionamento abreviado - Série Sementes, Circular Técnica, 56 ISSN 1516-7860, Londrina, PR Maio, 2008.

COSTA, P.R.; CUSTÓDIO, C.C.; MACHADO NETO, N.B.; MARUBAYASHI, O.M. Estresse hídrico induzido por manitol em sementes de soja de diferentes tamanhos. Revista Brasileira de Sementes, v.26, n.1, p.105-113, 2004. https://doi.org/10.1590/S0101-31222004000200015

DAN, Liana Lopes; Vera Delfina Colvara Mello; WETZEL, Clóvis Terra; POPINIGIS, Flavio; ZONTA, Élio Paulo; "Transferência de matéria seca como método de avaliação do vigor de sementes de soja." Revista Brasileira de Sementes 9.3 (1987): 45-55.

FRANÇA NETO, J.B.; KRZYZANOWSKI, F.C.; COSTA, N.P. da. 0 teste de tetrazólio em sementes de soja. Londrina: EMBRAPA-CNPSo, 1998. 72p. (EMBRAPA-CNPSo. Documentos, 116).

MARCOS FILHO, Junior; Fisiologia de sementes de plantas cultivadas; biblioteca de ciências agrarias Luiz de Queiroz, vol. 12; 495p; 2005.

MARCOS FILHO, Junior. Soja: Tecnologia da Produção. Avaliação da qualidade de sementes de soja. Piracicaba: Editor G. M. S. Câmara. p.206-243, 1998.

LIMA, R. M. "Efeito do tamanho das sementes sobre alguns atributos fisiológicos e agronômicos." Associação brasileira dos produtores de sementes: Anuário Abrasem, Brasília, DF (1996): 39-43.

MUNIZZI, A; BRACCINI.; A.L.; RANGEL, MA. S; SCAPIM; CA; ALBRECHT, L.P. Qualidade de sementes de quatro cultivares de soja, colhidas em dois locais no estado de Mato Grosso do Sul. Revista Brasileira de Sementes: v.32, n.1, p.176-185, 2010. https://doi.org/10.1590/S0101$\underline{31222010000100020}$

NAKAGAWA, J. Testes de vigor baseados no desempenho das plântulas. In: KRZYZANOWSKI, F.C.; VIEIRA, R.D.; FRANÇA NETO, J.B. (Ed.). Vigor de sementes: conceitos e testes. Londrina: ABRATES, 1999. cap.2.1, p.2.24.

PADILHA; Fábio Ronei Rodrigues; reconhecimento de variedades de soja através do Processamento de imagens digitais usando redes neurais

Artificiais; ljuí, RS, 2007. Programa de pós-graduação em modelagem matemática; Unijuí Universidade Regional Do Noroeste Do Estado Do Rio Grande Do Sul; 2007. 
PÁDUA, Gilda Pizzolante; ZITO, Roberto Kazuhiko; ARANTES, Neylson Eustáquio; FRANÇA NETO, José de Barros; Influência Do Tamanho Da Semente Na Qualidade Fisiológica E Na Produtividade Da Cultura Da Soja, Revista Brasileira de Sementes, vol. 32, no 3 p. 009-016, 2010

SALUM, Juliane Dossi. "Testes laboratoriais para a avaliação do desempenho germinativo no campo de sementes de lotes de soja (Glycine max (L.) Merrill." (2009): xiii-53.

SCHUCH, L.O.B.; NEDEL, J.L.; ASSIS, F.N. Crescimento em laboratório de plântulas de aveia-preta (Avena strigosa Schreb.) em função do vigor das sementes. Revista Brasileira de Sementes, v.21, n.1, p.229-234, 1999. https://doi.org/10.17801/0101-3122/rbs.v21n1p229-234

SOUZA, Lìlian Christian Domingues; Efeito da aplicação de fertilizante mineral via foliar sobre a produção e qualidade fisiológica de sementes de soja / Lìlian Christian Domingues de Souza. Ilha Solteira: [s.n.], $200751 \mathrm{f}$.

VANZOLINI, S.; CARVALHO, N.M. Efeito do vigor de sementes de soja sobre o seu desempenho em campo. Revista Brasileira de Sementes, v.24, n.1, p.33-41, 2002.

VINHAL-FREITAS, Isabel Cristina; JUNIOR ,José Edson Garcia; SEGUNDO Jurandir Pereira; VILARINHO, Muriel Silva; Germinação E Vigor De Sementes De Soja Classificadas Em Diferentes Tamanhos - Agropecuária Técnica - ISSN 0100-7467 - Areia, PB - CCA-UFPB v. 32, n. 1, 2011. 